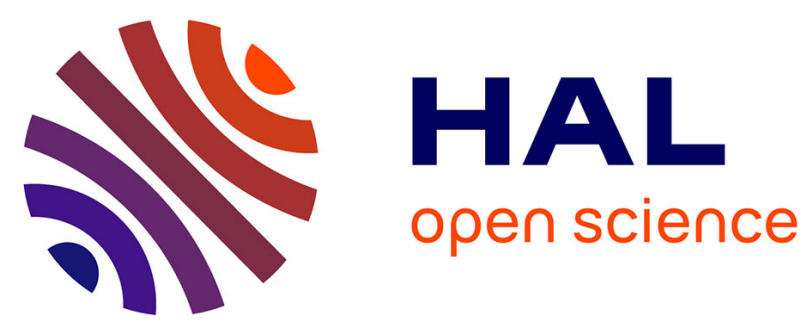

\title{
PERCEPTION DE HAUTEURS DE SONS DANS LE CAS DE SIGNAUX DE BRUIT MODULÉS PÉRIODIQUEMENT
}

\author{
J. Gruber
}

\section{- To cite this version:}

J. Gruber. PERCEPTION DE HAUTEURS DE SONS DANS LE CAS DE SIGNAUX DE BRUIT MODULÉS PÉRIODIQUEMENT. Journal de Physique IV Proceedings, 1992, 02 (C1), pp.C1-209C1-212. 10.1051/jp4:1992142 . jpa-00251213

\section{HAL Id: jpa-00251213 https://hal.science/jpa-00251213}

Submitted on 1 Jan 1992

HAL is a multi-disciplinary open access archive for the deposit and dissemination of scientific research documents, whether they are published or not. The documents may come from teaching and research institutions in France or abroad, or from public or private research centers.
L'archive ouverte pluridisciplinaire HAL, est destinée au dépôt et à la diffusion de documents scientifiques de niveau recherche, publiés ou non, émanant des établissements d'enseignement et de recherche français ou étrangers, des laboratoires publics ou privés. 


\title{
PERCEPTION DE HAUTEURS DE SONS DANS LE CAS DE SIGNAUX DE BRUIT MODULÉS PERIODIQUEMENT
}

\author{
J. GRUBER \\ Institut für Technische Akustik, Technische Universität Berlin, Einsteinufer 25, D-1000 Berlin 10, \\ Germany
}

Le son d'un bruit blanc modulé périodiquement a très souvent fait l'objet d'étude depuis la première recherche effectuée en 1948 par Miller et Taylor. Alors que quelques chercheurs (par ex., Miller et Taylor (1948), Harris (1963) ou Burns et Viemeister (1976) ont soutenu l'existence d'une perception sonore au moins faible aux fréquences de modulation basses, d'autres chercheurs la réfutent (par ex., Mowbray, Gebhard et Byham (1956) ou Meyer-Eppler, Sendhoff et Rupprath (1959)). L'importance théorétique de ce problème résuite du fait que le signal en question possède une fonction temporelle périodique évidente, le spectre de ce signal étant blanc. Un tel signal est un signal décisif pour étudier la question liant la structure temporelle d'un signal à la perception sonore de ce signal. Cette étude est centrée sur le fait que la modulation d'un bruit blanc ne produit un véritable spectre blanc que si le bruit devant être modulé est lui-même véritablement blanc. De simples petits écarts avec une fonction spectrale plate idéale conduisent à des périodicités dans le spectre du signal de bruit modulé. De tels périodicités sont reliées à la fréquence de modulation. Bien que ce fait semble être plutôt trivial, il a du être mentionné dans les études précédentes puisqu'il semble qu'il soit la raison possible de la perception sonore observée quelquefois. Si l'on fait l'usage d'écarts drastiques avec le spectre blanc à moduler (par ex., bruit rose), on peut observer une perception sonore nette.

The pitch of periodically modulated white noise had been investigated very often since its first examination by Miller and Taylor in 1948. While some investigators (e.g. Miller and Taylor(1948), Harris (1963) or Burns and Viemeister (1976) had reported on at least a faint pitch perception at low modulation frequencies, other investigators do not support these findings (e.g. Mowbray, Gebhard and Byham (1956) or Meyer-Eppler, Sendhoff and Rupprath (1959)). The theoretical importance of this problem results from the fact that the signal in question possesses a clear periodic time function while the spectrum is white. So gated noise is a crucial signal to investigate the question of a timestructure related pitch perception. This study is focussed on the fact that modulating a white noise only produces a really. white spectrum if the noise to be modulated also is really white. Just small deviations from an ideal flat spectral function lead to periodicities in the spectrum of the modulated noise signal. Such periodicities are related to the modulating frequency. Although this fact seems to be rather trivial, it should, however, have been mentioned in the former studies since it seems to be a possible reason for the sometimes observed pitch perception. If drastic deviations from the white spectrum to be modulated are used (e.g. pink noise) a clear pitch perception can be observed. 


\section{Perception de bruits modulés}

Les phénomènes de perception de bruits modulés ont été décrits en détail pour la première fois en 1948 par A.Miller et W.G.Taylor /1/. Ils ont modulé un bruit blanc avec une tension du type fonction-rectangle. Le "rapport on-off" utilisé était de 1:1. Le spectre d'un bruit blanc modulé est de nouveau blanc. Cependant, sa perception est différente: pour des fréquences de modulation de quelques $\mathrm{Hz}$, quelques impulsions de bruit se succèdant rapidement peuvent être entendues; au-dessus d'une fréquence de modulation de $20 \mathrm{~Hz}$ environ, on a certes l'existence d'une perception sonore continue, celle-ci étant cependant plus rugueux que celle du bruit continu. La différence entre un bruit modulé et un bruit continu disparait seulement à partir d'une fréquence de modulation de $1000 \mathrm{~Hz}$ environ. En outre, Miller et Taylor ont constatè qu'une perception sonore existe jusqu'à une fréquence de modulation d'environ $300 \mathrm{~Hz}$. Toutefois, ce fait est peu clair: "the pitch is vague, rough and diffuse..."

Cet effet est plein d'importance pour la théorie de la perception sonore car il ne peut s'expliquer que par une analyse de fonctions temporelles, le spectre ne contenant pas l'information de hauteur de tons. Un tel mécanisme nécessite de plus une soi-disant "horloge interne» qui, d'un point de vue physiologique, ne se représente pas bien. Par conséquent, il n'est pas étonnant que cette expérience ait été répétée constamment tout au long des décennies après 1948. Les résultats se sont contredits: Mowbray, Gebhard et Byham /2/ de même que Meyer-Eppler, Sendhoff et Rupprath /3/ et que Gruber /4/ n'ont pas pu confirmer ce phénomène de perception sonore démontré. D'autres auteurs, au contraire, ont confirmé la perception sonore de bruits modulés: Harris /5/, Pollack /6/, Walliser /7/, Burns et Viemeister /8/. Au cours de leurs travaux, Pollack, de même que Burns et Viemeister ont trouvé une limite supérieure de la fréquence de modulation jusqu'à laquelle un son est perçu $(800 \mathrm{~Hz}$ environ chez Burns et Viemeister, et jusqu'à $2000 \mathrm{~Hz}$ chez Pollack).

\section{Sources d'artefact possibles}

\subsection{Modulateur "non soigné»}

Mowbray et Hart /2/ ont indiqué un effet évident d'artefact: des parties spectrales de la tension de modulation qui peuvent, dans le cas d'un modulateur mal équilibré, pénétrer dans le signal modulé. Lors du contôle très consciencieux du modulateur, on ne pouvait plus constater une perception sonore. Toutefois, d'autres auteurs (Harris, Pollack) ont considéré ce problème et l'ont soigneusement écarté.

\subsection{Spectres de signaux de bruit modulés}

Une autre source d'artefact n'ayant jusqu'à présent jamais été évoquée se trouve au niveau des spectres des signaux de bruits modulès. Le bruit modulé est associé à un spectre blanc à l'unique condition que le bruit, avant la modulation, soit véritablement blanc. Dans le cas d'un spectre présentant au départ des écarts avec un véritable spectre blanc, des parties du spectre associé au signal modulateur apparaissent, lors de la modulation, dans le spectre du signal modulé. Ces parties peuvent engendrer, suivant leur importance, une perception sonore.

\subsubsection{Spectre du bruit filtré par un passe-haut et modulé}

Pour beaucoup de sources de bruit, les fréquences les plus basses ne parviennent pas jusqu'à la sortie. Ainsi, on module un bruit filtré ayant une fréquence de coupure $f_{H P}$. On obtient le spectre du bruit modulé en convoluant les spectres de bruit avec les fonctions temporelles modulatrices: soit $m(f)$, le spectre du signal modulateur, $R(f)$, le spectre du bruit. Le spectre du signal modulé s'obtient donc par: $R_{m}(f)=m(f) \star R(f)$; (figure 1$)$. II apparaît, au niveau de la fréquence de modulation $f_{m}$, une rupture de spectre avec une profondeur de $3 \mathrm{~dB}$ environ et une largeur de bande de $2 f_{H P}$. Les autres ruptures appa- 


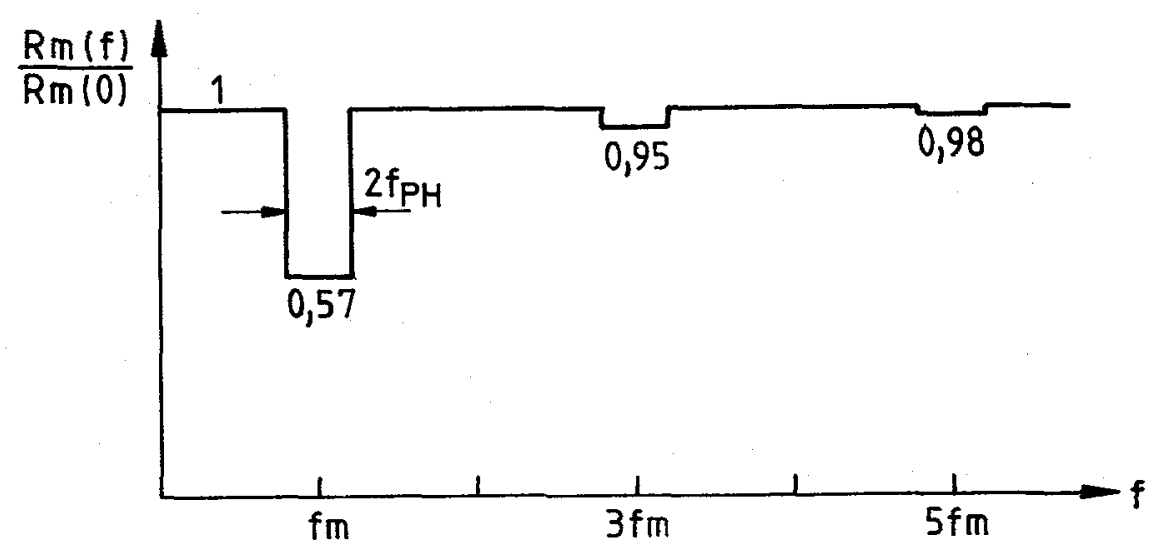

Fig. 1 Spectre du bruit filtré par un passe-haut et modulé

raissant aux fréquences $3 f_{m}, 5 f_{m} \ldots$ sont petites, sans importance. La rupture du spectre à la fréquence de modulation pourrait être la cause de la perception sonore constatée. Cette perception est peu nette car la rupture est petite. Pour des fréquences de passehaut fixées $f_{H P}$, la largeur de bande relative $f_{H P} / f_{m}$ de la rupture diminue pour des fréquences $f_{m}$ croissantes. Ceci pourrait expliquer le fait que les sons ont seulement pu être perçus pour des fréquences de modulation basses. Les observations personellement menées concernant les bruits filtrés par un passe-haut et modulés confirment cette hypothèse.

\subsubsection{Spectre du bruit filtré par un passe-bas et modulé}

Figure 2 montre le spectre d'un bruit filtré par un passe-bas et modulé, au sein duquel on a choisi $f_{P B}>>f_{m}$. On peut également penser que ce cas constitue une source d'artefact. Dans la partie supérieure du spectre, il apparait des degrés dont la largeur de bande est un multiple de $f_{m}$. Ces degrés conduisent de la même façon à une perception sonore du signal, cette fois cependant pas à une hauteur de ton correspondant à la fréquence de modulation $f_{m}$.

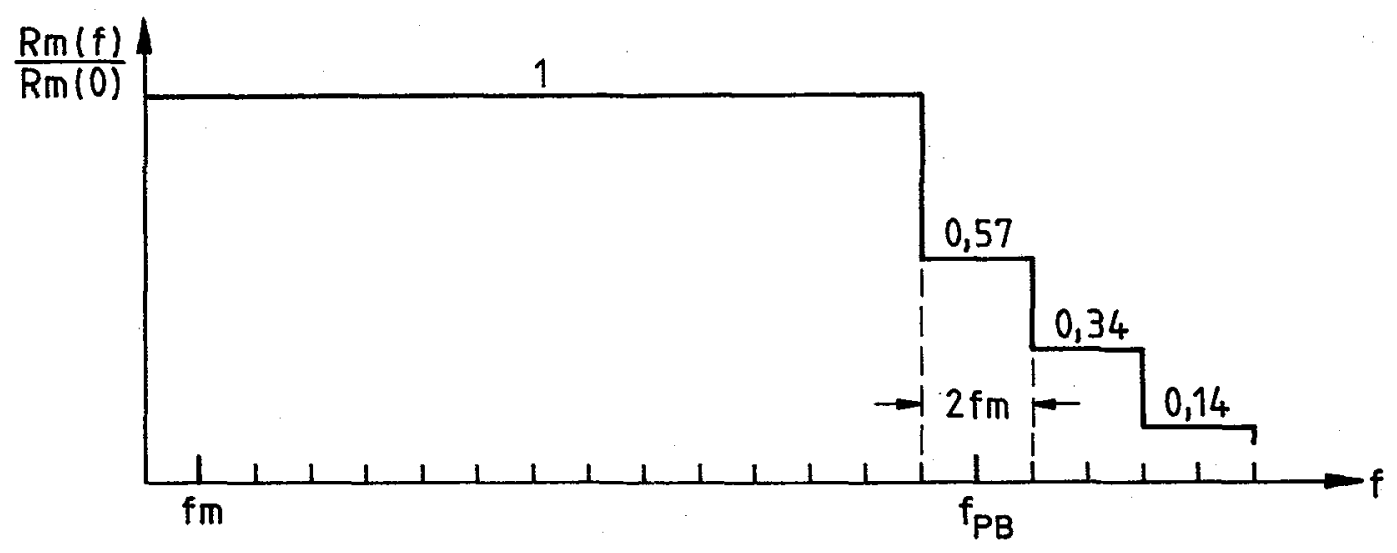

Fig.2 Spectre du bruit filtré par un passe-bas et modulé 


\subsubsection{Spectre d'un bruit rose modulé}

Figure 3 montre le spectre d'un bruit rose modulé (pente de $-3 d B / o c t$.$) . Là apparaissent$ des pics spectraux aux fréquences $f_{m}, 3 f_{m} \ldots$; le spectre ressemble au spectre en forme de peigne. Le bruit rose modulé a une tonalité bien forte et ce, également dans le cas de fréquences de modulation élevées. La tonalité faiblit avec la diminution de la pente du spectre du bruit modulé et disparait lorsque cette pente se situe au sein de l'intervalle 0,5 - 1dB/oct, dans la mesure où le "rapport on-off" de la fonction rectangle modulatrice est de 1:1. La tonalité est d'autant plus marquée que le urapport on-off» est petit; le spectre en forme de peigne est alors d'autant plus marqué. Il apparait alors des pics aux fréquences $2 f_{m}, 4 f_{m} \ldots$ Du fait que de petis écarts avec le spectre blanc conduisent à une perception sonore, il peut être tenu pour possible que ce spectre modulé en forme de peigne constitue une source d'artefact.

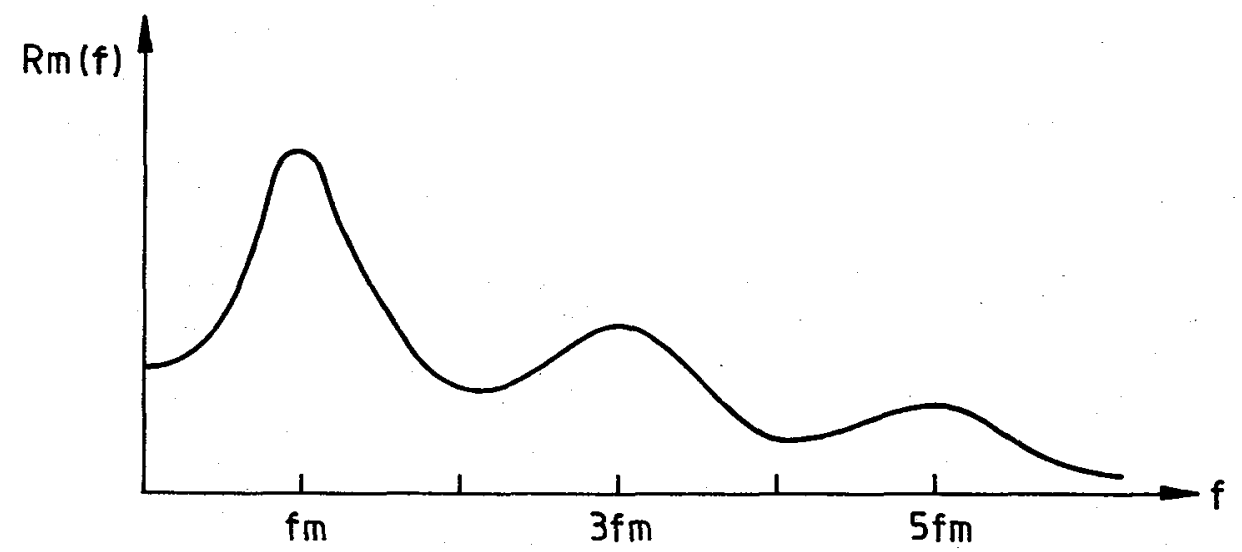

Fig.3 Spectre du bruit rose modulé

/1/ Miller, G.A., Taylor, W.G., J.Acoust.Soc.Am.20.171 (1948).

12/ Mowbray, G.H., Gebhard, J.W., Byham, C.L., J.Acoust.Soc.Am.28.106 (1956).

/3/ Meyer-Eppler, W.,Sendhoff,H., Rupprath, R.., Gravesaner Blätter 14.70 (1959).

/4/ Gruber, J., Diss. TU Berlin D83 (1967).

/5/ Harris, G.G., J.Acoust.Soc.Am.35.1229 (1963).

/6/ Pollack, I., J.Acoust.Soc.Am.45.237 (1969).

/7/ Walliser, K., Acustica 21.329 (1969).

/8/ Burns, E.M., Viemeister, N.F., J.Acoust.Soc.Am.60.863 (1976). 